\title{
Focusing of Sound in a 3D Phononic Crystal
}

\author{
Suxia Yang, ${ }^{1,2, *}$ J. H. Page, ${ }^{1}$ Zhengyou Liu, ${ }^{3}$ M. L. Cowan,,${ }^{1,}$ C.T. Chan,${ }^{2}$ and Ping Sheng ${ }^{2}$ \\ ${ }^{1}$ Department of Physics and Astronomy, University of Manitoba, Winnipeg, Manitoba, Canada R3T 2N2 \\ ${ }^{2}$ Department of Physics, Hong Kong University of Science and Technology, Kowloon, Hong Kong, China \\ ${ }^{3}$ Department of Physics, Wuhan University, Wuhan 430072, China
}

(Received 1 March 2004; published 7 July 2004)

\begin{abstract}
We present a combined experimental and theoretical study of phonon focusing phenomena in a pass band above the complete band gap in a 3D phononic crystal. Wave propagation was found to depend dramatically on both frequency and incident direction. This propagation anisotropy leads to very large negative refraction, which can be used to focus a diverging ultrasonic beam into a narrow focal spot with a large focal depth. The experimental field patterns are well explained using a Fourier imaging technique, based on the 3D equifrequency surfaces calculated from multiple scattering theory.
\end{abstract}

DOI: 10.1103/PhysRevLett.93.024301

PACS numbers: 43.35.+d, 63.20.-e

The past decade has witnessed a rapid growth in the study of phononic crystals [1-12], which are periodic composite materials that are the elastic and acoustic analogues of photonic crystals [13-21]. This growing interest is fueled not only by potential applications as novel acoustic devices [7,8,10-12], but also by the rich physics governing elastic and acoustic wave propagation in periodic media [1-12]. In addition, ultrasonic and acoustic techniques, coupled with powerful theoretical approaches, provide some unique advantages for directly investigating wave phenomena in these systems. Most of the studies until now have focused on the existence and properties of phononic band gaps [1-7,9], which occur due to Bragg scattering when the wavelength is comparable with the lattice constants, leading to frequency bands where wave propagation is forbidden. The result has been considerable progress in understanding how to achieve large complete band gaps in physically realizable materials, and in elucidating the mechanism of wave transport at gap frequencies, which has been shown to occur by tunneling [9].

However, relatively little attention has been paid to investigating how periodicity affects wave propagation over a wide range of frequencies outside the band gaps, where novel refractive, diffractive, and focusing effects may all be possible. At low frequencies, an effective medium or continuum approximation can be adopted to study the wave properties and accurately predict the wave speeds. In this frequency range, there is much in common with the properties of low frequency phonons in atomic crystals, where phonon focusing phenomena have been systematically studied [22]. Recently, low frequency 2D sonic crystal refractive acoustic devices for airborne sound have been demonstrated [10] and theoretically analyzed [11] at wavelengths well below the first acoustic band gap. Also, a theory for tailoring sonic devices with dimensions on the order of several wavelengths has been investigated, where image formation was shown to occur predominantly through a diffraction mechanism rather than by refraction [12]. By contrast, much less is known about the behavior at higher frequencies in pass bands where the wavelengths can be much less than the lattice constant. In this Letter, we address this question both theoretically and experimentally by investigating the characteristics of wave scattering and propagation in a 3D phononic crystal at frequencies above the first complete band gap. We show how a dramatic variation in wave propagation with both frequency and propagation direction leads to novel focusing phenomena associated with large negative refraction. We study these effects experimentally using ultrasonic techniques to image the transmitted wave field, and demonstrate that a flat crystal can focus a diverging incident beam into a sharp focal spot that can be seen remarkably far from the crystal. The field patterns are calculated theoretically using a Fourier imaging technique, in which wave propagation through the crystal is accurately described by the $3 \mathrm{D}$ equifrequency surfaces predicted from the multiple scattering theory (MST) [4]. Our theoretical results give an excellent explanation of the experimental data, showing how wave physics in this regime can be accurately modeled and allowing potential applications to be developed based on the study of the equifrequency surfaces of phononic crystals.

Our phononic crystals consist of 0.800-mm-diameter tungsten carbide beads surrounded by water, with the beads close packed in a face centered cubic crystal structure along the body diagonal or [111] crystal direction $(\Gamma \mathrm{L})$. The large acoustic mismatch for this combination of materials gives rise to a complete band gap for frequencies from 0.98 to $1.2 \mathrm{MHz}$ [9]. Using the newly developed MST [3,4], we calculated not only the band structure $[\omega(k)$, where $\omega$ is the angular frequency, $k$ is the wave vector $\left(k=\omega / v_{p}\right)$ and $v_{p}$ is the phase velocity], but also the $3 \mathrm{D}$ dispersion or equifrequency surface at each frequency in the first Brillouin zone (BZ). The equifrequency surface, which is a graphical representation of the variation in the magnitude of the wave vector with 
direction for a particular frequency, is the key to understanding the effect of anisotropy on wave propagation in phononic crystals. In particular, the equifrequency surface (frequently called the slowness surface in acoustics) allows the direction of energy transport to be identified for each $k$, since this direction is determined by the group velocity $v_{g}$, which is perpendicular to the surface [ $v_{g}=$ $\left.\nabla_{k} \omega(k)\right]$. Figure 1(a) shows cross sections of the reduced zone equifrequency surfaces in a crystal plane defined by the high symmetry points $\Gamma, \mathrm{X}, \mathrm{L}, \mathrm{K}$. This range of frequencies near $1.6 \mathrm{MHz}$ is interesting, since the equifrequency surfaces are strongly anisotropic and frequency dependent, and there is only one branch in the band structure [9]. Also, in the first Brillouin zone, the group velocity is positive for directions near [111] only when $k$ and $v_{p}$ are negative; for isotropic equifrequency surfaces, this condition has been taken to indicate left-handed behavior in photonic crystals [19], by analogy to lefthanded materials in which both the permittivity and permeability are negative [23]. An equivalent representation of the wave vector anisotropy, which may be more physically appealing, can be obtained by translating the equifrequency surfaces to the extended zone scheme [Fig. 1(b)], in which the wave vector magnitudes correspond to those measured from the cumulative phase shift in a transmission experiment [24]. Near $1.6 \mathrm{MHz}$ in directions around the $\Gamma \mathrm{L}$ direction, the translation formula is $\vec{k} \rightarrow \vec{k}+2 \vec{G}_{111}$, where $\vec{G}_{111}$ is the shortest reciprocal vector along the $\Gamma \mathrm{L}$ direction. Note that, in the extended zone scheme, both $v_{g}$ and $k$ are positive. In both the reduced and the extended zone schemes, the anisotropy of the equifrequency surfaces causes the group velocity to point back towards the $\Gamma \mathrm{L}$ direction as the wave vector moves away from this direction, suggesting that both novel focusing and large negative refraction phenomena may occur.

To explore this question in detail, we measured wave propagation through a 12-layer crystal with a thickness of $7.985 \mathrm{~mm}$. Figure 2(a) gives a schematic diagram of the

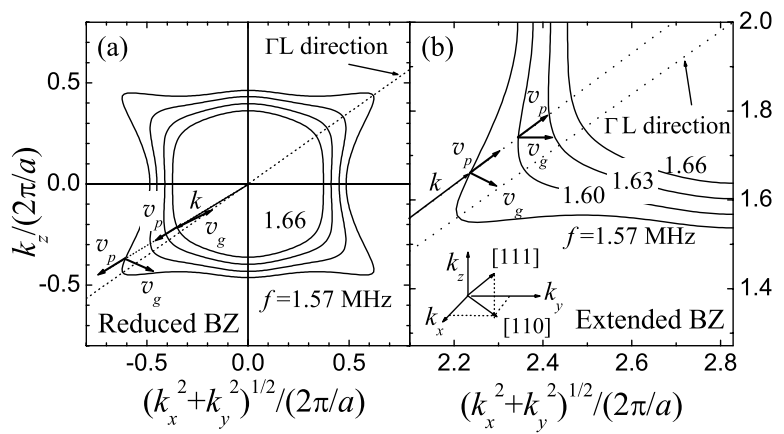

FIG. 1. Cross sections of the equifrequency surfaces at frequencies near $1.60 \mathrm{MHz}$ in the reduced (a) and extended Brillouin zones (b). The cross sections are shown in a plane containing the [001], [111], and [110] directions, as shown in the inset. experimental setup, showing the position of the phononic crystal and its supporting substrate with respect to the generating ultrasonic transducer and the detecting hydrophone. The entire assembly was immersed in water. As the generating transducer, we used a pinducer, which has a small cross sectional area (radius $\sim$ wavelength, $\lambda$ ) and is therefore a good approximation to a point source. The pinducer was placed close to the sample surface $(3 \mathrm{~mm}$ away). The pulse transmitted through the sample and substrate was detected using a miniature hydrophone, which had a diameter much less than the ultrasonic wavelength. The hydrophone was mounted on a 3D translation stage, allowing the field to be detected at each point of a square grid in the horizontal plane $3 \mathrm{~mm}$ below the bottom of the substrate. The pulses were then transformed into the frequency domain using a fast-Fourier transform (FFT) technique, allowing the wave amplitude for each frequency component to be plotted as a function of position in the detecting plane.

To investigate the effect of the crystal on wave propagation, we first recorded the reference wave field measured through the bare substrate. The results at 1.57 and 1.60 MHz are plotted in Figs. 3(a1) and 3(b1), respectively. It can be seen that the amplitude of the reference wave field diminishes gradually as the detecting point moves away from the middle, as expected for the far field radiation pattern of the small diameter disk-shaped transducer used in these experiments. By contrast, the measured field patterns with the crystal in place (at the same two frequencies) are shown in Figs. 3(a2) and 3(b2); they are seen to be completely different. At $1.57 \mathrm{MHz}$, the wave is focused into a tight spot with a FWHM of $5 \mathrm{~mm}$ (about five wavelengths). At $1.60 \mathrm{MHz}$, however, the incident beam is spread out by the crystal; the field pattern shows a ring structure and exhibits threefold symmetry along the $\Gamma \mathrm{L}$ direction. Thus, the transmitted field pattern changes completely with only a $2 \%$ change in frequency.
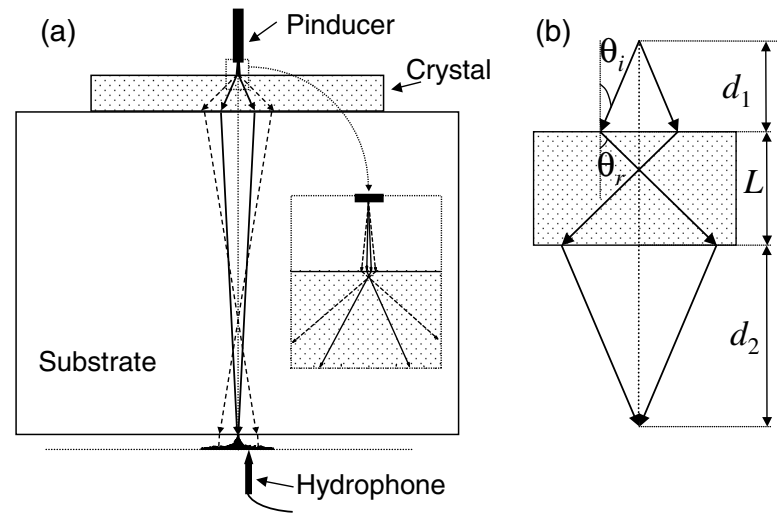

FIG. 2. (a) Experimental setup, with rays indicating the predicted directions of the group velocity at $1.6 \mathrm{MHz}$ for angles of incidence of $1.5^{\circ}$ and $5^{\circ}$. (b) Diagram showing the focusing condition in a medium with negative refraction. 

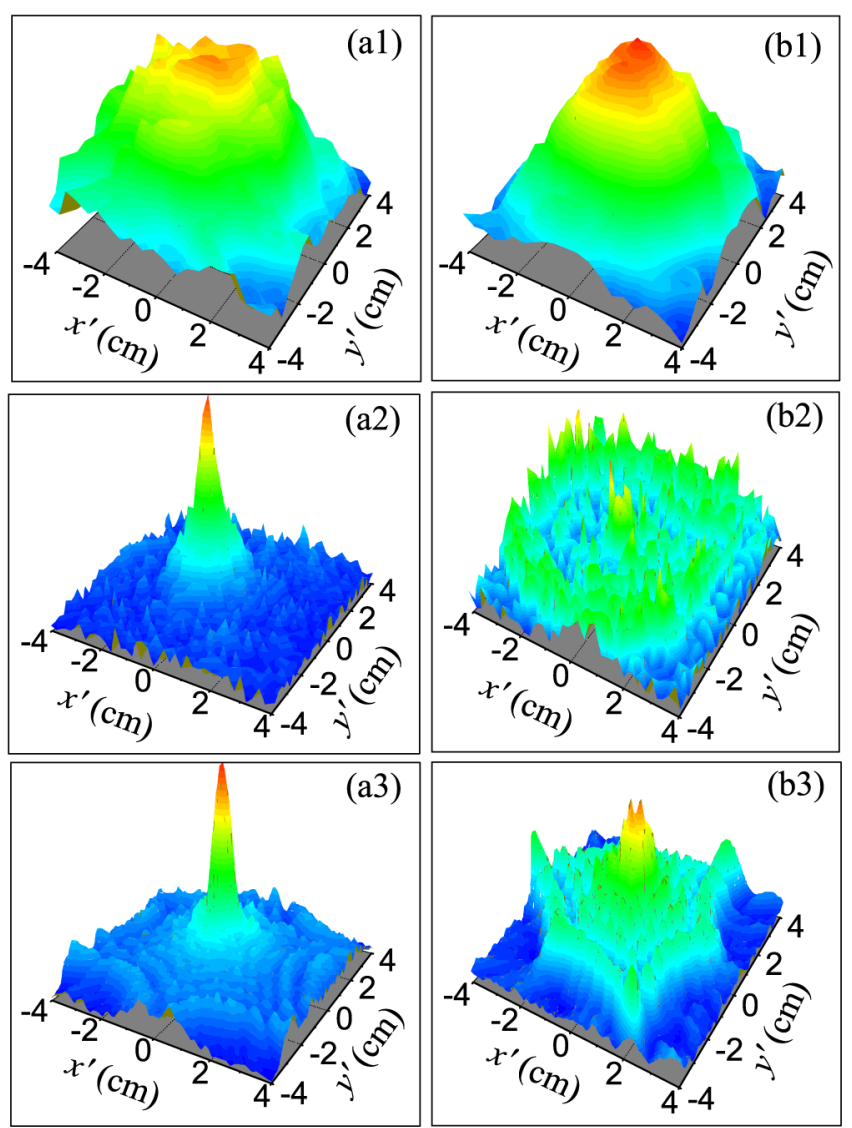

FIG. 3 (color online). Left side: Field patterns measured at $1.57 \mathrm{MHz}$ without (a1) and with (a2) the crystal in place, along with the theoretical prediction (a3). The $x^{\prime}$ and $y^{\prime}$ axes are in the plane parallel to the sample surface, with $x^{\prime}$ in the LK direction. Right side: Corresponding field patterns for measurements at $1.60 \mathrm{MHz}$.

To interpret our data, we calculated the wave field patterns using a Fourier imaging technique. For a given frequency, the key to this calculation is to determine the phase difference between all the waves arriving at each point in the detecting plane after the waves have propagated through the sample, substrate and water [Fig. 2(a)]. At the input surface of the sample, the incident wave field was first Fourier transformed from real space into the spatial frequency domain, essentially decomposing it into plane waves at each frequency. This enabled the boundary conditions at each interface to be readily implemented, since it was then straightforward to ensure, for each constituent plane wave, that the component of wave vector parallel to the interface be conserved (Snell's law). Propagation through the crystal was then described by using the crystal's 3D dispersion relation to determine the resulting phase shift of each of the constituent plane waves. The outgoing waves were then allowed to propagate in a normal way through the substrate and water. At the detection plane, the wave field was transformed back to real space by taking the inverse FFT. The results at frequencies of 1.60 and $1.63 \mathrm{MHz}$ are plotted in
Figs. 3(a3) and 3(b3). We can see that the theory gives an excellent prediction of the behavior observed experimentally, although the frequency is shifted up by $2 \%$ at both frequencies $(1.57 \rightarrow 1.60 \mathrm{MHz}$ and $1.60 \rightarrow$ $1.63 \mathrm{MHz}$ ). A similar frequency shift is found between theoretical predictions and experimental measurements of the nearby band-edge frequency, indicating that, relative to the band edge, there is excellent agreement between theory and experiment. As seen in the experimental data, the theory predicts the energy to be strongly focused at the lower frequency. At the slightly higher frequency, however, the energy is defocused and the symmetry in the field pattern is clearly seen.

To further study the focusing effect at the lower frequency, we also measured and calculated the cross section of the field pattern in a plane perpendicular to the crystal layers. The experimental and theoretical results are plotted in Figs. 4(a1) and 4(a2), respectively, and show the extension of the focal zone as a function of distance (measured in wavelengths $\lambda$ ) away from the substrate. The theory and data are in reasonable agreement. Because of the thick substrate, we were not able to measure the field pattern closer to the crystal surface, so we have used our theoretical model to investigate the focusing behavior in this region. These theoretical predictions are shown in Fig. 4(b), where it can be seen that the distance from the
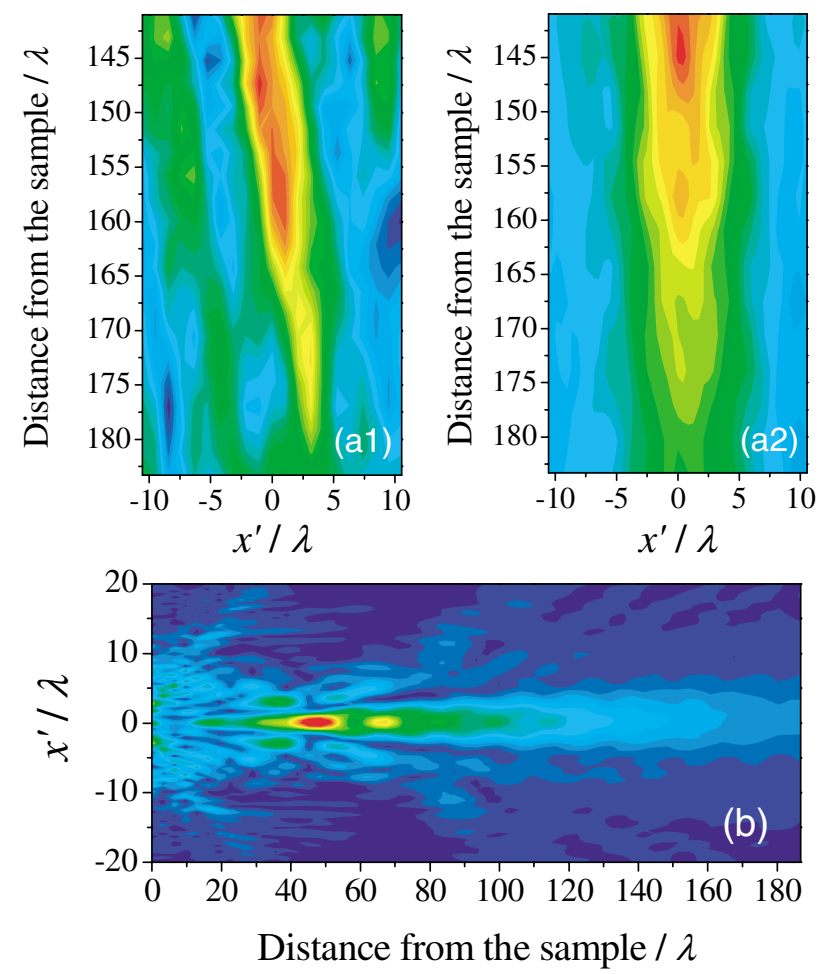

FIG. 4 (color online). Measured (a1) and calculated (a2) cross sections of the field pattern in a plane perpendicular to the crystal layers for measurements at $1.57 \mathrm{MHz}$. The bottom figure (b) shows the calculated focal pattern closer to the crystal surface. 
surface of the crystal where the focal spot is sharpest (the analogue of focal length) is $47 \lambda$ ( $44 \mathrm{~mm}$ in water at $1.60 \mathrm{MHz}$ ). There the diameter of the focal spot (at $-6 \mathrm{~dB}$ ) is $2.4 \lambda$, close to the diffraction limit. Figure 4(b) also shows that the average depth of the focal zone is quite long (about 50 $\lambda$ ), but there is also a large quasiperiodic modulation of the pattern as the distance increases, with a periodicity of $\sim 15 \lambda$. Near the sample $(z<30 \lambda)$, the field pattern is quite complex, but still shows the characteristic threefold symmetry expected about the [111] direction.

Additional insight into the formation of the focusing pattern shown in Figs. 3 and 4 can be obtained by tracing ray diagrams that indicate the directions of the group velocity for different angles of incidence. This is illustrated in Fig. 2(a) for two representative angles of incidence, calculated at a frequency of $1.6 \mathrm{MHz}$. Inside the crystal, the beam follows the direction of the group velocity and bends the "wrong" way, appearing to undergo negative refraction even though the wave vectors of each plane wave component obey Snell's law with positive (normally refracted) angles of incidence and refraction. As shown in the inset of Fig. 2(a), the apparent angles of refraction $\theta_{r}$ are large and negative (e.g., for an angle of incidence $\theta_{i}=2.0^{\circ}, \theta_{r}=-30^{\circ}$ ), leading to an initial ray crossing just inside the crystal, and followed by a second focus on the far side of the crystal. To illustrate the physics of the focusing further, we have drawn a ray diagram in Fig. 2(b) for the case where the medium on either side of the crystal is the same. This shows that a point source is imaged at the position $d_{1}+$ $d_{2}=\beta L$, where $L$ is the thickness of the phononic crystal, $d_{1}$ and $d_{2}$ are the distances from the source and imaging points to the front and back interfaces, respectively, and $\beta=\left|\tan \theta_{r} / \tan \theta_{i}\right|$ is a parameter that is determined by the magnitude of the apparent negative refraction angle relative to the direction of the incident wave vector. Thus, when the source is moved closer to the crystal, the image on the other side moves further away, but there is no central axis in the system: Moving the source point parallel to the interface moves the image point by the same amount. We note that $\beta$ depends on both frequency and incident angle in our case; thus, the imaging depends on frequency, and the angular dependence results in a shaped image with a long focal zone.

In summary, we have demonstrated both experimentally and theoretically how phononic crystals can "bend" ultrasound beams in unusual ways due to the anisotropy of the equifrequency surfaces in phononic crystals. The crystal can be used to focus a diverging sound beam without the curved interfaces used in conventional lenses, with a focal length that can be varied by varying the frequency. In addition to delineating the basic physics, the excellent agreement between our theoretical model and experiment lays a theoretical framework for engineering phononic crystal lenses and prisms, ranging from acoustic superlenses with ideal focusing characteristics to 024301-4 beam-shaping lenses with long focal zones, such as the example considered above. As all the properties of phononic crystal are scalable, the study of such bending effects may also provide new tools for improving sound beams.

Support from NSERC of Canada, RGC (HKUST6143/ 00P) of Hong Kong, and NSFC (10174054) is gratefully acknowledged.

*Current address: Dept. of Materials Science Eng., Univ. of Toronto, Toronto, ON, Canada M5S 3E4.

${ }^{\dagger}$ Current address: Department of Physics, Univ. of Toronto, Toronto, ON, Canada M5S 3E3.

[1] E. N. Economou and M. M. Sigalas, Phys. Rev. B 48, 13434 (1993); J. Acoust. Soc. Am. 95, 1734 (1994).

[2] M. S. Kushwaha et al., Phys. Rev. Lett. 71, 2022 (1993); Eur. Phys. J. B 3, 155 (1998).

[3] M. Kafesaki and E. N. Economou, Phys. Rev. B 60, 11993 (1999); I. E. Psarobas, N. Stefanou, and A. Modinos, Phys. Rev. B 62, 278 (2000).

[4] Z. Liu et al., Phys. Rev. B 62, 2446 (2000).

[5] Z. Liu et al., Science 289, 1734 (2000).

[6] M. Torres, F. R. Montero de Espinosa, and J. L. Aragón, Phys. Rev. Lett. 86, 4282 (2001).

[7] J. O. Vasseur et al., Phys. Rev. Lett. 86, 3012 (2001).

[8] J.H. Page et al., in Photonic Crystals and Light Localization in the 21st Century, edited by C.M. Soukoulis (Kluwer Academic, Amsterdam, 2001), p. 59.

[9] S. Yang et al., Phys. Rev. Lett. 88, 104301 (2002); Phys. Canada 57, 187 (2001).

[10] F. Cervera et al., Phys. Rev. Lett. 88, 023902 (2002).

[11] B. C. Gupta and Z. Ye, Phys. Rev. E 67, 036603 (2003).

[12] N. Garcia et al., Phys. Rev. E 67, 046606 (2003).

[13] E. Yablonovitch, Phys. Rev. Lett. 58, 2059 (1987).

[14] S. John, Phys. Rev. Lett. 58, 2486 (1987).

[15] Photonic Band Gaps and Localization, edited by C. M. Soukoulis (Plenum, New York, 1992).

[16] J. D. Joannopoulos, R. D. Meade, and J. N. Winn, Photonic Crystals (Princeton University Press, Princeton, 1995).

[17] H. Kosaka et al., Phys. Rev. B 58, 10096 (1998); Appl. Phys. Lett. 74, 1212 (1999).

[18] M. Notomi, Phys. Rev. B 62, 10696 (2000); J. Mizuguchi et al., Phys. Rev. B 67, 075109 (2003).

[19] S. Foteinopoulou, E. N. Economou, and C. M. Soukoulis, Phys. Rev. Lett. 90, 107402 (2003); E. Cubukcu et al., Nature (London) 423, 604 (2003).

[20] P.V. Parimi et al., Nature (London) 426, 404 (2003).

[21] C. Luo et al., Phys. Rev. B 68, 045115 (2003).

[22] J. P. Wolfe, Imaging Phonons: Acoustic Wave Propagation in Solids (Cambridge University Press, Cambridge, England, 1998).

[23] J. B. Pendry, Phys. Rev. Lett. 85, 3966 (2000).

[24] Note that, in an ultrasonics experiment, the detectors are phase sensitive, allowing the cumulative phase $k L$ to be determined unambiguously after propagation through a sample of thickness $L$. This can be achieved by measuring the phase as a function of frequency down to low enough frequencies that the phase shift is less than $2 \pi$. 